\title{
GAUGE INDEPENDENT HAMILTONIAN REDUCTION OF CONSTRAINED SYSTEMS
}

\section{S. I. MUSLIH}

Received 31 October 2001 and in revised form 18 March 2002

A gauge independent method of obtaining the reduced space constrained dynamics is discussed in a purely Hamiltonian formalism. Three examples are studied.

\section{Introduction}

One of the important aspects of the Hamiltonian formulation of gauge theories is to obtain the reduced (physical) space comprising the total canonical variables. This is usually done by fixing a gauge that removes the unphysical degrees of freedom $[3,10]$.

Fixing a gauge is not always an easy task, especially when first-class constraints arise, which are generators of gauge transformations, this will lead to the gauge freedom. In other words, the equations of motion are still degenerate and depend on the functional arbitrariness. We have to impose external gauge fixing constraint for each first-class constraint.

To avoid the ambiguities and arbitrariness inherent in the gauge fixing produced, it becomes desirable to abstract the reduced space in a gauge independent manner. Recently, the Hamilton-Jacobi approach [8, $9,12,13,15]$ has been developed to investigate singular systems. In this method, the equations of motion are obtained as total differential equations in many variables. If the system is integrable then we can obtain directly the canonical reduced phase-space coordinates. The advantages using the Hamilton-Jacobi is that we have no difference between firstand second-class constraints and we do not need gauge fixing term because the gauge variables are separated in the process of constructing an integrable system of total differential equations. 
On the other hand, there are approaches other than gauge fixing. For instance, the method of Faddeev and Jackiw [4] and Jackiw [11] is to attempt to reduce the system to its physical degrees of freedom by a process of directly substituting the constraints into the canonical Lagrangian. Besides, Banerjee [1, 2] has developed a purely Lagrangian approach of systematically reducing the degrees of freedom in a gauge independent manner. The physical Hamiltonian is then obtained directly from this reduced Lagrangian.

In this paper, the Hamilton-Jacobi treatment for three singular systems will be discussed to obtain the reduced phase space dynamics in a gauge independent manner.

\section{Lagrangian and Hamiltonian approaches}

In this section, we briefly review the Lagrangian and the Hamiltonian formulations for studying the constrained systems.

\subsection{Lagrangian approach}

Recently, Banerjee $[1,2]$ has developed a purely Lagrangian approach to obtain the reduced degree of freedom in a gauge independent manner. Now, we give a brief review of his method.

From the theory of unsolvable differential equations with respect to the highest derivatives, it is possible to express the Lagrangian equations of second-order systems with variables $v$ by an equivalent set of independent equations $[1,2]$,

$$
\begin{aligned}
\ddot{p} & =\Theta(p, \dot{p}, q, \beta, \dot{\beta}, \ddot{\beta}), \\
\dot{q} & =\Phi(p, \dot{p}, q, \beta, \dot{\beta}), \\
r & =\Psi(p, q, \beta),
\end{aligned}
$$

where $v=(p, q, r, \beta), \Theta, \Phi, \Psi$ are some functions of the indicated arguments and an overdot denotes a time derivative. In a nonsingular theory, $q, r, \beta$ are absent so that there are unconstrained dynamics with $\ddot{p}=$ $\Theta(p, \dot{p})$. For singular theories, (2.2) and (2.3) represent the constraints. The idea is now to pass from the constrained $v=(p, q, r, \beta)$ to the unconstrained $v=p$ by removing $q, r, \beta$. The variable $r$ is trivially eliminated in favour of $p, q, \beta$ using (2.3). In the physically interesting gauge systems, the constraints are implemented by a Lagrange multiplier whose time derivative, therefore, does not appear in the Lagrangian. This multiplier is identified with $q$, which can thus be removed in favour of $p, \beta$ using (2.2). The Lagrangian in the reduced sector is now a function of $(v, \dot{v} ; v=p, \beta)$. By evaluating the Lagrange equations in this sector, it is 
possible to identify $\beta$ with the variable that does not occur in these equations. With this identification the variable $\beta$, which reflects the degeneracy in the system, automatically drops out from the Lagrangian and its final unconstrained form is obtained. The physical Hamiltonian is now found by performing the standard Legendre transformation without using any gauge fixing conditions.

\subsection{The Hamilton-Jacobi approach}

The starting point of the Hamilton-Jacobi method $[8,9,12,13,15]$ is to consider the Lagrangian $L=L\left(q_{i}, \dot{q}_{i}, t\right), i=1, \ldots, n$, with the Hessian matrix

$$
A_{i j}=\frac{\partial^{2} L}{\partial \dot{q}_{i} \partial \dot{q}_{j}}, \quad i, j=1, \ldots, n,
$$

of rank $(n-r), r<n$. Then $r$ momenta are dependent. The generalized momenta $p_{i}$ corresponding to the generalized coordinates $q_{i}$ are defined as

$$
\begin{aligned}
& p_{a}=\frac{\partial L}{\partial \dot{q}_{a}}, \quad a=1,2, \ldots, n-r, \\
& p_{\mu}=\frac{\partial L}{\partial \dot{x}_{\mu}}, \quad \mu=n-r+1, \ldots, n,
\end{aligned}
$$

where $q_{i}$ are divided into two sets, $q_{a}$ and $x_{\mu}$. Since the rank of the Hessian matrix is $(n-r)$, we solve (2.5) for $\dot{q}_{a}$ as

$$
\dot{q}_{a}=\dot{q}_{a}\left(q_{i}, \dot{x}_{\mu}, p_{a} ; t\right)
$$

Substituting (2.7) into (2.6), we get

$$
p_{\mu}=-H_{\mu}\left(q_{i}, \dot{x}_{\mu}, p_{a} ; t\right)
$$

The canonical Hamiltonian $H_{0}$ reads

$$
H_{0}=p_{a} \dot{q}_{a}+\left.p_{\mu} \dot{x}_{\mu}\right|_{p_{v}=-H_{v}}-L\left(t, q_{i}, \dot{x}_{v}, \dot{q}_{a}\right), \quad \mu, v=n-r+1, \ldots, n
$$

The set of Hamilton-Jacobi partial differential equations (HJPDE) is expressed as

$$
H_{\alpha}^{\prime}\left(x_{\beta}, q_{a}, \frac{\partial S}{\partial q_{a}}, \frac{\partial S}{\partial x_{\alpha}}\right)=0, \quad \alpha, \beta=0, n-r+1, \ldots, n,
$$




\section{Gauge independent Hamiltonian reduction}

where

$$
H_{0}^{\prime}=p_{0}+H_{0}, \quad H_{\mu}^{\prime}=p_{\alpha}+H_{\mu}
$$

we define $p_{\beta}=\partial S\left[q_{a} ; x_{\alpha}\right] / \partial x_{\beta}$, and $p_{a}=\partial S\left[q_{a} ; x_{\alpha}\right] / \partial q_{a}$ with $x_{0}=t$ and $S$ being the action. The equations of motion are obtained as total differential equations in many variables as follows [8,9]:

$$
\begin{gathered}
d q_{a}=\frac{\partial H_{\alpha}^{\prime}}{\partial p_{a}} d x_{\alpha}, \quad d p_{a}=-\frac{\partial H_{\alpha}^{\prime}}{\partial q_{a}} d x_{\alpha}, \quad d p_{\beta}=-\frac{\partial H_{\alpha}^{\prime}}{\partial x_{\beta}} d x_{\alpha,} \\
d z=\left(-H_{\alpha}+p_{a} \frac{\partial H_{\alpha}^{\prime}}{\partial p_{a}}\right) d x_{\alpha,}
\end{gathered}
$$

where $z=S\left(x_{\alpha} ; q_{a}\right)$. These equations are integrable if and only if $[12,13]$

$$
d H_{0}^{\prime}=0, \quad d H_{\mu}^{\prime}=0, \quad \mu=n-r+1, \ldots, n .
$$

If conditions (2.14) are not satisfied identically, we consider them as new constraints and again consider their variations. Thus, repeating this procedure, we may obtain a set of constraints such that all the variations vanish. Simultaneous solutions of canonical equations with all these constraints produce the set of canonical phase space coordinates $\left(q_{a}, p_{a}\right)$ as functions of $x_{\alpha}$; the canonical action integral is obtained in terms of the canonical coordinates. The Hamiltonian $H_{\alpha}^{\prime}$ can be interpreted as the infinitesimal generators of canonical transformations given by parameters $x_{\alpha}$, respectively.

\section{Examples}

To illustrate the above ideas given in Section 2, we will solve three singular systems by the Lagrangian method [1, 2], and then by the HamiltonJacobi method $[8,9,12,13,15]$.

\subsection{The Christ-Lee model}

As a first example, consider the motion of a particle in two-dimensional space whose dynamics are governed by the Lagrangian $[7,14]$

$$
L\left(x_{i}, \dot{x}_{i}, q\right)=\frac{1}{2} \dot{x}_{i}^{2}-\epsilon_{i j} x_{i} \dot{x}_{i} q+\frac{1}{2} q^{2} x_{i}^{2}-V\left(\rho^{2}\right),
$$

where $x_{i}=x_{1}, x_{2}$ are the rectilinear coordinates of the dimensional vector $\vec{\rho}$ so that $x_{i}^{2}=x_{1}^{2}+x_{2}^{2}=\rho^{2}$ and $q$ is another coordinate. The antisymmetric tensor $\epsilon_{i j}$ is defined such that $\epsilon_{12}=1$. 
Now we investigate this model using the Lagrangian approach. The Lagrangian equations of motion are given by [2]

$$
\ddot{x}_{i}+2 \epsilon_{i j} q \dot{x}_{j}+\epsilon_{i j} \dot{q} x_{j}-q^{2} x_{i}+\frac{\partial V}{\partial x_{i}}=0, \quad q \rho^{2}-\epsilon_{i j} x_{i} \dot{x}_{j}=0 .
$$

To obtain the reduced Lagrangian, the coordinate $q$ is now eliminated from (3.1) using (3.2) to yield

$$
L_{r}\left(x_{i}, \dot{x}_{i}\right)=\frac{\left(x_{i} \dot{x}_{i}\right)^{2}}{2 x_{j}^{2}}-V\left(\rho^{2}\right) .
$$

Introducing the polar decomposition

$$
x_{1}=\rho \cos \phi, \quad x_{2}=\rho \sin \phi,
$$

we obtain the unconstrained Lagrangian

$$
L(\rho, \dot{\rho})=\frac{1}{2} \dot{\rho}^{2}-V\left(\rho^{2}\right)
$$

in terms of the physical variable $\rho$.

The reduced Hamiltonian is calculated as

$$
H\left(\rho, \pi_{\rho}\right)=\pi_{\rho} \dot{\rho}-L(\rho, \dot{\rho})=\frac{1}{2} \pi_{\rho}^{2}+V\left(\rho^{2}\right)
$$

where $\pi_{\rho}=\dot{\rho}$ is the momenta conjugated to $\rho$.

We next consider the Hamilton-Jacobi $[8,9,12,13,15]$ treatment of the Christ-Lee model. The generalized momenta read as

$$
\pi_{i}=\dot{x}_{i}+q \epsilon_{i j} x_{j}, \quad \pi_{q}=0 .
$$

Since the rank of the Hessian is two, we have only one primary constraint as

$$
H_{q}^{\prime}=\pi_{q}=0
$$

The canonical Hamiltonian reads

$$
H_{0}=\frac{1}{2} \pi_{i}^{2}+q \epsilon_{i j} \pi_{i} x_{j}+V\left(\rho^{2}\right)
$$

Following the Hamilton-Jacobi method, we obtain the set of HJPDE as

$$
H_{0}^{\prime}=p_{0}+\frac{1}{2} \pi_{i}^{2}+q \epsilon_{i j} \pi_{i} x_{j}+V\left(\rho^{2}\right)=0, \quad H_{q}^{\prime}=\pi_{q}=0 .
$$


114 Gauge independent Hamiltonian reduction

Imposing

$$
d H_{q}^{\prime}=0
$$

leads to the constraint

$$
H_{1}^{\prime}=\epsilon_{i j} \pi_{i} x_{j}=0
$$

The total variation of $H_{1}^{\prime}$ is identically zero and no further constraints arise. Hence, we obtain the canonical Hamiltonian as

$$
H^{c}=\left.H_{0}\right|_{H_{1}^{\prime}=0}=\frac{1}{2} \pi_{i}^{2}+V\left(\rho^{2}\right) .
$$

Now making use of the following canonical transformations $[1,2,7$, 14]:

$$
\begin{array}{ll}
x_{1}=\rho \cos \phi, & x_{2}=\rho \sin \phi, \\
\pi_{1}=\pi_{\rho} \cos \phi-\frac{\pi_{\phi}}{\rho} \sin \phi, & \pi_{2}=\pi_{\rho} \sin \phi+\frac{\pi_{\phi}}{\rho} \cos \phi,
\end{array}
$$

and taking into account constraint (3.12), we obtain the canonical reduced Hamiltonian,

$$
H_{c}^{r}=\left.H_{0}\right|_{H_{1}^{\prime}=0}=\frac{1}{2} \pi_{\rho}^{2}+V\left(\rho^{2}\right)
$$

The equivalence between (3.15) and (3.6) shows the gauge independent way of obtaining the reduced Hamiltonian in terms of the physical phase space $\left(\rho, \pi_{\rho}\right)$.

The corresponding action integral has the following form:

$$
z=\int d t\left[\pi_{\rho} \dot{\rho}-\frac{1}{2} \pi_{\rho}^{2}-V\left(\rho^{2}\right)\right]
$$

\subsection{The extended Christ-Lee model}

As a second example, we investigate the extended Christ-Lee model [5], which is an extension of model (3.1), where we introduce a third Cartesian coordinate $z$, such that the new Lagrangian reads $[2,5]$

$$
L\left(x_{i}, \dot{x}_{i}, \dot{z}, q\right)=\frac{1}{2} \dot{x}_{i}^{2}-g \epsilon_{i j} x_{i} \dot{x}_{i} q+\frac{1}{2} g^{2} q^{2} x_{i}^{2}+\frac{1}{2}(\dot{z}-q)^{2}-V\left(x_{1}^{2}+x_{2}^{2}\right)
$$

where $g>0$ is a coupling parameter. 
As in the previous example, we obtain the reduced Hamiltonian for the model (3.17) using first the Lagrangian approach and then the Hamilton-Jacobi approach.

The Lagrangian equations of motion read as

$$
\begin{gathered}
\ddot{x}_{i}+2 \epsilon_{i j} g q \dot{x}_{j}+\epsilon_{i j} \dot{q} g x_{j}-q^{2} g^{2} x_{i}+\frac{\partial V}{\partial x_{i}}=0, \quad \ddot{z}-\dot{q}=0, \\
g^{2} q x_{i}^{2}-g \epsilon_{i j} x_{i} \dot{x}_{j}-\dot{z}+q=0 .
\end{gathered}
$$

Equation (3.19) can be solved to obtain

$$
q=\frac{\dot{z}+g\left(\epsilon_{i j} x_{i} \dot{x}_{j}\right)}{1+g^{2}\left(x_{1}^{2}+x_{1}^{2}\right)} .
$$

Eliminating $q$ from the Lagrangian (3.17) using (3.20) and the polar decomposition (3.4), we obtain the reduced Lagrangian [2]

$$
L=\frac{1}{2}\left[\dot{\rho}^{2}+\left(1+g^{2} \rho^{2}\right)^{-1}(\dot{\phi}-g \dot{z})^{2}\right]-V\left(\rho^{2}\right) .
$$

In order to obtain the reduced Hamiltonian, we introduce the new variable

$$
\Phi=\phi-g z
$$

in this case the reduced Lagrangian is obtained in terms of the unconstrained variables as

$$
L=\frac{1}{2}\left[\dot{\rho}^{2}+\left(1+g^{2} \rho^{2}\right)^{-1}(\dot{\Phi})^{2}\right]-V\left(\rho^{2}\right) .
$$

Now the canonically conjugated momenta are given by

$$
\pi_{\rho}=\dot{\rho}, \quad \pi_{\Phi}=\frac{\rho^{2}}{1+g^{2} \rho^{2}} \dot{\Phi} .
$$

The reduced Hamiltonian is obtained in terms of the canonical variables $\left(\rho, \pi_{\rho}\right)$ and $\left(\Phi, \pi_{\Phi}\right)$ as

$$
H=\frac{1}{2} \pi_{\rho}^{2}+\frac{1}{2}\left(g^{2}+\frac{1}{\rho^{2}}\right) \pi_{\Phi}^{2}+V\left(\rho^{2}\right) .
$$

We now follow the Hamilton-Jacobi method [8, 9, 12, 13, 15] to investigate the extended Christ-Lee model. The set of Hamilton-Jacobi partial 
116 Gauge independent Hamiltonian reduction

differential equations is given by

$$
H_{0}^{\prime}=p_{0}+H_{0}=0, \quad H_{q}^{\prime}=\pi_{q}=0,
$$

where $H_{0}$ is given by

$$
H_{0}=\frac{1}{2}\left(\pi_{i}^{2}+\pi_{z}^{2}\right)+q\left(\pi_{z}+g \epsilon_{i j} x_{i} \pi_{j}\right)+V\left(\rho^{2}\right) .
$$

Imposing the integrability condition,

$$
d H_{q}^{\prime}=0,
$$

we obtain the constraint

$$
H_{1}^{\prime}=\pi_{z}+g \epsilon_{i j} x_{i} \pi_{j}=0
$$

The total variation of $H_{1}^{\prime}$ is identically zero and no further constraints arise. Making use of (3.29), the canonical reduced Hamiltonian is given by

$$
H_{c}^{r}=\left.H_{0}\right|_{H_{1}^{\prime}=0}=\frac{1}{2}\left[\pi_{1}^{2}\left(1+g^{2} x_{2}^{2}\right)+\pi_{2}^{2}\left(1+g^{2} x_{1}^{2}\right)-2 g^{2} x_{1} x_{2} \pi_{1} \pi_{2}\right]+V\left(\rho^{2}\right) .
$$

We should notice the equivalence of this Hamiltonian, modulo canonical transformations, with expression (3.25). These transformations are given by $[1,2,14]$

$$
\begin{array}{rlrl}
x_{1} & =\rho \cos \Phi, & x_{2} & =\rho \sin \Phi, \\
\pi_{1}=\pi_{\rho} \cos \Phi-\frac{\pi_{\Phi}}{\rho} \sin \Phi, & \pi_{2}=\pi_{\rho} \sin \Phi+\frac{\pi_{\Phi}}{\rho} \cos \Phi
\end{array}
$$

Again, the equivalence between (3.30) and (3.25) shows the gauge independent way of obtaining the reduced Hamiltonian in terms of the canonical phase space $\left(\rho, \pi_{\rho}\right)$ and $\left(\Phi, \pi_{\Phi}\right)$.

Now, the action integral is calculated by making use of (2.13), (3.30), and (3.31) as follows:

$$
z=\int d t\left[\pi_{\rho} \dot{\rho}+\pi_{\Phi} \dot{\Phi}-\frac{1}{2} \pi_{\rho}^{2}-\frac{1}{2}\left(g^{2}+\frac{1}{\rho^{2}}\right) \pi_{\Phi}^{2}-V\left(\rho^{2}\right)\right] .
$$




\subsection{Electromagnetic theory}

As a third example, we consider the free electromagnetic theory as a constrained system. The Lagrangian density is given by

$$
\mathcal{L}=-\frac{1}{4} F^{\mu v} F_{\mu \nu}, \quad \mu \nu=0,1,2,3
$$

We discuss the Lagrangian treatment of this model. The equations of motion are

$$
\partial_{\mu} F^{\mu v}=0
$$

For $v=0$, we have the constraint

$$
\partial^{2} A_{0}+\partial_{0}\left(\partial_{i} A^{i}\right)=0
$$

The multiplier $A_{0}$ can be eliminated in favour of the other variables by solving constraint (3.35). Using this, we express (3.33) in terms of the reduced variables. The Lagrange equations in these variables are $[1,2,6]$

$$
\partial^{j} F_{j i}+\partial_{0}^{2}\left[\left(\delta_{i j}-\frac{\partial_{i} \partial_{j}}{\partial^{2}}\right) A_{j}\right]=0
$$

Now choosing the orthogonal polarization:

$$
A_{i}=A_{i}^{T}+A_{i}^{L}
$$

the reduced Lagrangian is obtained as

$$
\mathcal{L}=\frac{1}{2} \dot{A}_{i}^{T^{2}}-\frac{1}{4} F_{i j}^{T}\left(A^{T}\right)
$$

Denoting the independent components of $A_{i}^{T}$ by $a_{I}(I=1,2)$;

$$
A_{i}^{T}=\left(\delta_{i I}-\delta_{i 3} \frac{\partial_{I}}{\partial_{3}}\right) a_{I}
$$

the Lagrangian (3.33) is expressed in terms of independent variables $a_{I}$. Taking the Legendre transformation of this Lagrangian, we obtain the reduced Hamiltonian,

$$
H_{0}^{r}=\int d^{3} x\left(\frac{1}{2}\left[\left(\delta_{i I}-\frac{\partial_{i} \partial_{I}}{\partial^{2}}\right) \pi_{I}\right]^{2}+\frac{1}{4} F_{i j}^{2}(a)\right) .
$$


118 Gauge independent Hamiltonian reduction

Now, we would like to investigate the electromagnetic theory of Maxwell using the Hamilton-Jacobi method. The momenta conjugated to the field $A^{\mu}$ are

$$
\pi^{\mu}=\frac{\partial \mathcal{L}}{\partial \dot{A}_{\mu}}=F^{\mu 0}
$$

Therefore,

$$
\pi^{0}=0
$$

is the primary constraint. The canonical Hamiltonian $H_{0}$ is expressed as

$$
H_{0}=\int d^{3} x\left(\frac{1}{4} F^{i j} F_{i j}+\frac{1}{2} \pi^{2}+\pi^{i} \partial_{i} A_{0}\right) .
$$

Starting from this Hamiltonian and making use of (2.11), the set of Hamilton-Jacobi partial differential equations reads as

$$
\begin{array}{ll}
H_{0}^{\prime}=\pi_{4}+H_{0}=0, & \pi_{4}=\frac{\partial S}{\partial t} \\
H^{\prime}=\pi_{0}=0, & \pi_{0}=\frac{\partial S}{\partial A_{0}} .
\end{array}
$$

Imposing $d \pi_{0}=0$, we obtain the constraint

$$
\partial_{i} \pi^{i}=0
$$

The total variation of constraint (3.45) is identically zero and no further constraints arise.

Making use of the following transformations $[1,6]$ :

$$
A_{i}^{T}=\left(\delta_{i I}-\delta_{i 3} \frac{\partial_{I}}{\partial_{3}}\right) a_{I}, \quad \pi_{i}^{T}=\left(\delta_{i I}-\frac{\partial_{i} \partial_{I}}{\partial^{2}}\right) \pi_{I},
$$

and using constraint (3.45), the Hamiltonian $H_{0}$ gets further reduced to

$$
H_{0}^{r}=\int d^{3} x\left(\frac{1}{2} \pi^{T^{2}}+\frac{1}{4} F_{i j}^{T^{2}}\right)
$$


where $\pi_{i}^{T}$ are the canonical momenta conjugated to $A_{i}^{T}$ and they satisfy the following Poisson bracket relation:

$$
\left\{A_{i}^{T}(x), \pi_{i}^{T}(y)\right\}=\delta_{i j} \delta^{3}(\vec{x}-\vec{y}) .
$$

The canonical action integral is calculated as

$$
z=\int\left\{d^{3} x\left(\pi_{I} \dot{a}_{I}-\frac{1}{2}\left[\left(\delta_{i I}-\frac{\partial_{i} \partial_{I}}{\partial^{2}}\right) \pi_{I}\right]^{2}-\frac{1}{4} F_{i j}^{2}(a)\right)\right\} d t
$$

\section{Conclusion}

In this work, we have investigated three constrained systems using the Hamilton-Jacobi approach [8, 9, 12, 13, 15]. In this approach, the equations of motion are obtained as total differential equations in many variables. If the system is integrable, then we can obtain the canonical reduced Hamiltonian in terms of the canonical physical variables without using any gauge fixing condition.

In the Christ-Lee model (3.1), using suitable canonical transformations (3.14) and taking into account the integrability conditions, we obtain the reduced Hamiltonian in terms of canonical physical variables $\left(\rho, \pi_{\rho}\right)$ without using any gauge fixing conditions. The second example is an extension of the Christ-Lee model. Again, using the canonical transformations and taking into account the integrability conditions we determine the physical Hamiltonian in a gauge independent manner.

The third example is the electromagnetic theory of Maxwell, which is an instructive example of singular continuous systems. This theory is invariant under gauge transformations of the second kind, that is,

$$
A_{\mu} \longrightarrow A_{\mu}+\partial_{\mu} F
$$

where $F$ is an arbitrary function. Therefore, the nonobservable quantity $A_{\mu}$ is not unique. The Hamilton-Jacobi method is used to reduce the degrees of freedom and to obtain the physical Hamiltonian for this system without using any gauge fixing condition.

For the three examples, the results obtained using the HamiltonJacobi method are in complete agreement with those obtained by the Lagrangian method $[1,2]$.

\section{Acknowledgment}

The author would like to thank the reviewers for their interesting remarks. 


\section{References}

[1] R. Banerjee, Gauge independent Lagrangian reduction of constrained systems, preprint, 1996, http:/ / arxiv.org/abs/hep-th/9607199.

[2] __ Gauge independent reduction of a solvable model with Gribov-like ambiguity, preprint, 1996, http:/ / arxiv.org/abs/hep-th/9610240.

[3] P. A. M. Dirac, Lectures on Quantum Mechanics, Academic Press, New York, 1964.

[4] L. Faddeev and R. Jackiw, Hamiltonian reduction of unconstrained and constrained systems, Phys. Rev. Lett. 60 (1993), 1692-1694.

[5] R. Friedberg, T. D. Lee, Y. Pang, and H. C. Ren, A soluble gauge model with Gribov-type copies, Ann. Physics 246 (1996), no. 2, 381-445.

[6] D. M. Gitman and I. V. Tyutin, Quantization of Fields with Constraints, Springer-Verlag, Berlin, 1990.

[7] S. A. Gogilidze, A. M. Khvedelidze, and V. N. Pervushin, On abelianization of first class constraints, J. Math. Phys. 37 (1996), no. 4, 1760-1771.

[8] Y. Güler, Canonical formulation of singular systems, Nuovo Cimento B (11) 107 (1992), no. 12, 1389-1395.

[9] _ Integration of singular systems, Nuovo Cimento B (11) 107 (1992), no. $10,1143-1149$.

[10] A. Hanson, T. Regge, and C. Teitelboim, Constrained Hamiltonian Systems, Accademia Nazionale dei Lincei, Rome, 1976.

[11] R. Jackiw, (Constrained) quantization without tears, Constraint Theory and Quantization Methods (Montepulciano, 1993), World Science Publishing, New Jersey, 1994, pp. 163-175.

[12] S. I. Muslih and Y. Güler, On the integrability conditions of constrained systems, Nuovo Cimento B (11) 110 (1995), no. 3, 307-315.

[13] _ Is gauge fixing of constrained systems necessary? Nuovo Cimento B 113 (1998), no. 3, 277-414.

[14] L. V. Prokhorov and S. V. Shabanov, Phase space of mechanical systems with a gauge group, Uspekhi Fiz. Nauk 161 (1991), no. 2, 13-75.

[15] E. Rabei and Y. Güler, Hamilton-Jacobi treatment of second-class constraints, Phys. Rev. A 46 (1992), 3513-3515.

S. I. Muslih: Department of Physics, Al-Azhar University, Gaza, Palestine E-mail address: sami_muslih@hotmail.com 


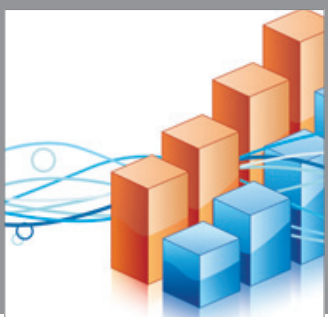

Advances in

Operations Research

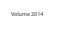

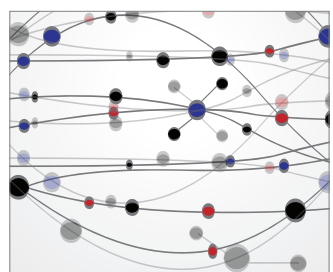

\section{The Scientific} World Journal
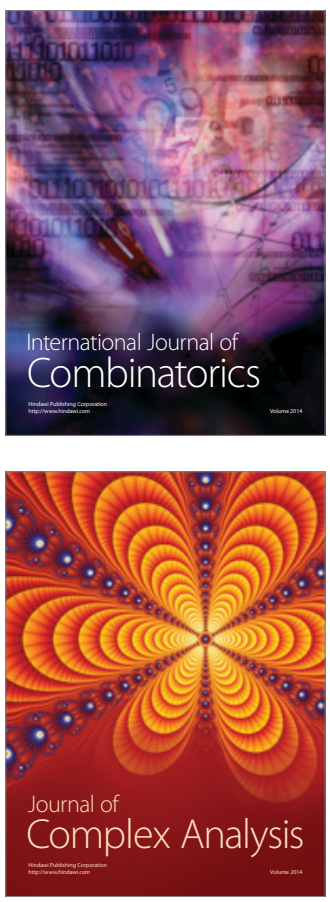

International Journal of

Mathematics and

Mathematical

Sciences
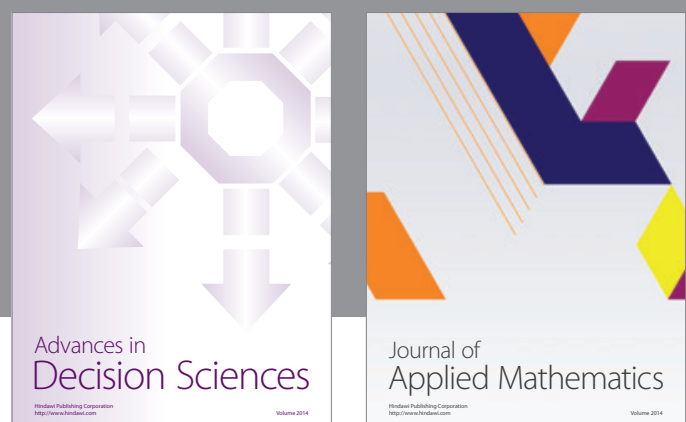

Journal of

Applied Mathematics
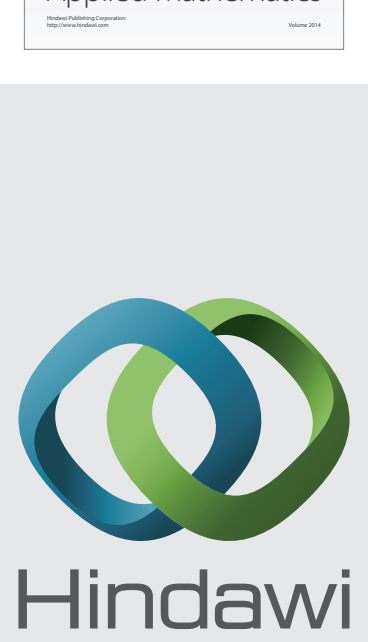

Submit your manuscripts at http://www.hindawi.com
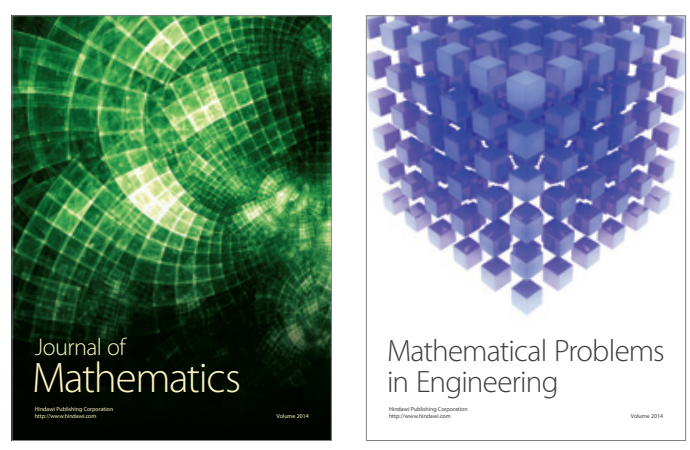

Mathematical Problems in Engineering
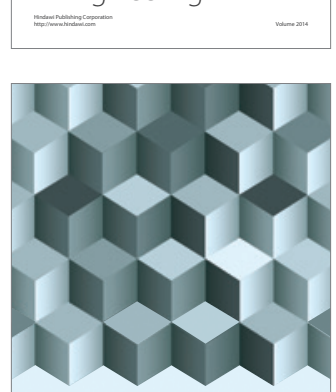

Journal of

Function Spaces
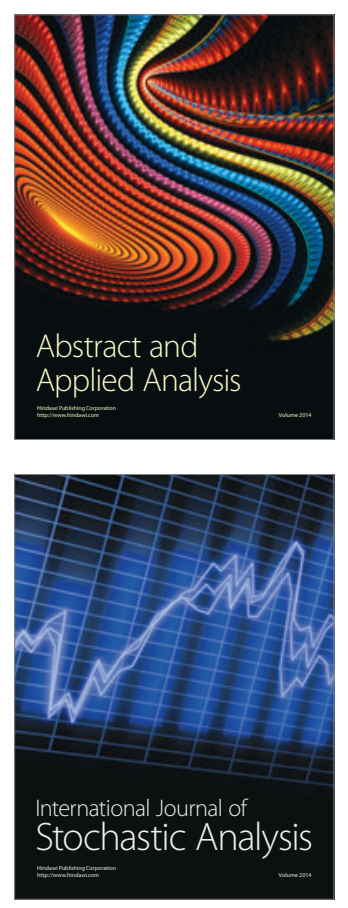

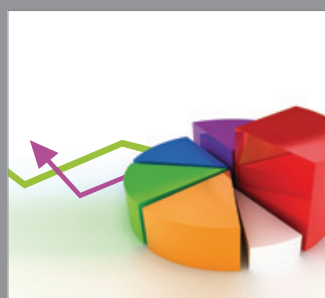

ournal of

Probability and Statistics

Promensencen
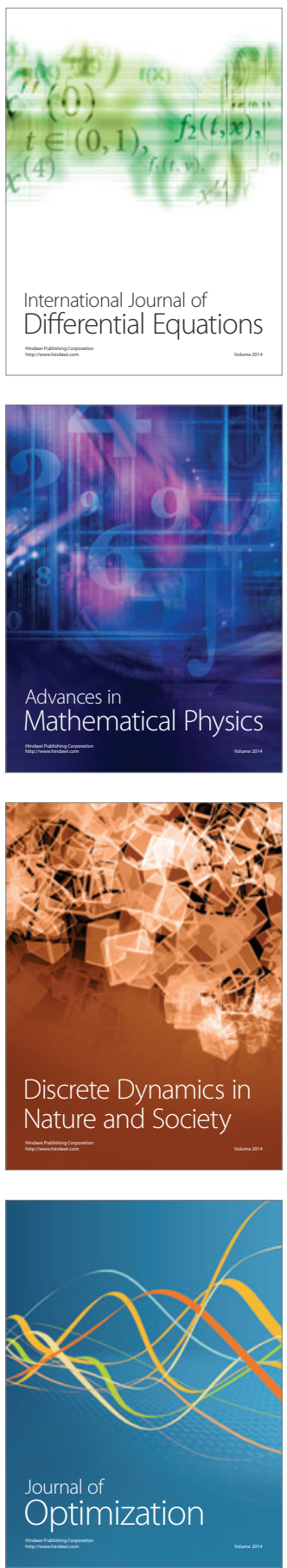